\title{
Negative air ion exposure ameliorates depression- like behaviors induced by chronic mild stress in mice
}

\section{Yunqing $\mathrm{Hu}$}

Fudan University

Tingting Niu

Lanzhou University

Jianming $\mathbf{X u}$

Shanghai Meteorological Bureau

Li Peng

Shanghai Meteorological Bureau

Qinghua Sun

Zhejiang Chinese Medical University

\section{Ying Huang}

Fudan University

Ji Zhou

Fudan University

Yuqiang Ding ( $\sim$ dingyuqiang@vip.163.com )

Fudan University

\section{Research Article}

Keywords: negative ions, air ions, depression, CMS, inflammation, cortisol

Posted Date: November 12th, 2021

DOI: https://doi.org/10.21203/rs.3.rs-974691/v1

License: (c) (1) This work is licensed under a Creative Commons Attribution 4.0 International License. Read Full License

Version of Record: A version of this preprint was published at Environmental Science and Pollution Research on April 11th, 2022. See the published version at https://doi.org/10.1007/s11356-022-20144-x. 


\section{Abstract}

The presence of negative air ions (NAl) is suggested to be a good factor in improving psychological status and used in treating depression as an alternative approach. However, the biological explanation for effects of NAI on alleviating depression symptoms has less been explored. In this study, the chronic mild stress (CMS) protocol was used to induce transcriptional depressive-like behaviors in mice, and the effects of NAl exposure on CMS-induced depression-like behaviors were examined. Thirty-day NAI exposure prevented the CMS-induced depression-like behaviors as shown by the restoration of sucrose preference and reduced immobility time in the suspension test. In addition, the elevation of serous corticosterone was present in CMS-treated mice but not existed in those with the NAl exposure. Furthermore, we observed a shifted balance between the cytokines secreted by type $1 \mathrm{~T}$ helper (Th1) cells and type $2 \mathrm{~T}$ helper (Th2) cells. In conclusion, NAI intervention is able to ameliorate CMS-induced depression-like behaviors in mice, and this effect is associated with the alteration of corticosterone and functional rebalance between Th1 and Th2 cells.

\section{Introduction}

Air ions are molecules of ionized particles present in the atmosphere, either losing or gaining an electric charge. They are generated in a variety of natural or artificial ways, such as rain, wind, snow, lightning, and ion generators, and currently are available for either domestic or industrial uses (Jiang, Ma, \& Ramachandran, 2018). It has been speculated that exposure to positive air ions in the environment is harmful to human health, while exposure to negative air ions (NAI) has beneficial health effects. Several explorations have focused on the biological effects of air ions on mood and behaviors (Bachman, McDonald, \& Lorenz, 1966; Della Vecchia, Mucci, \& Marazziti, 2020; Olivereau, Lambert, \& Truongngoc, 1981). A meta-analysis reported that the exposure to the air ions show no consistent results on the performances of wheel running, spontaneous locomotion, brain electrical activity and sleep patterns in animals (Bailey, Williams, \& Leonhard, 2018), while other studies measuring the effects of air ionization on various psychological parameters related to mood or emotional state have demonstrated that higher concentrations of NAl exposure is positively associated with mental health (Chu et al., 2019; Jiang et al., 2018; Perez, Alexander, \& Bailey, 2013).

The etiology of depression is complex and diverse (Duman, Aghajanian, Sanacora, \& Krystal, 2016; Krishnan \& Nestler, 2008). Etiological hypotheses of this disorder include the dysfunctions of monoaminergic system, hyperactivity of hypothalamic-pituitary-adrenal (HPA) axis, inflammatory alterations and neurotrophic abnormalities (Villas Boas et al., 2019). Hyperactivity of HPA axis shown by high serous level of cortisol is present in a large population of depressed patients (Hinkelmann et al., 2009; Leonard, 2018), and also observed in animal models (X. Q. Wang et al., 2021; Zaletel, Filipovic, \& Puskas, 2016). Cortisol is released in response to stress and regulates immune and inflammatory processes, energy metabolism and neuronal survival (Nikkheslat, Pariante, \& Zunszain, 2018; Zunszain, Anacker, Cattaneo, Carvalho, \& Pariante, 2011). Increasing data have evidenced that inflammation and 
HPA axis hyperactivity often coexist in the episodes of depression (Cernackova, Durackova, Trebaticka, \& Mravec, 2020; Gold, 2015).

Inflammation has been shown to interact with almost all pathophysiological domains known to be related to depression (Kim, Na, Myint, \& Leonard, 2016; A. H. Miller, V. Maletic, \& C. L. Raison, 2009). Cytokines are polypeptides or glycoproteins synthesized and secreted by peripheral monocytes, macrophages, lymphocytes, and as well as brain cells such as neurons, astrocytes and microglia. They play important roles in the bidirectional immune communication between the brain and the periphery (Leonard, 2018). Specifically, cytokines produced in the periphery can access and together with those generated within the brain influence the function state of the brain through humoral and neural pathways (Capuron \& Miller, 2011). They are critical in the immune response to help to mark and eliminate the pathogens, and also affect many aspects of brain functions such as neurotransmitter metabolism and its activity, neuroendocrine, neurogenesis, and neurocircuits relevant to mood, alarm, anxiety and motor activity (Himmerich, Patsalos, Lichtblau, Ibrahim, \& Dalton, 2019). Previous studies have found that the administration of interferon- $\gamma$ (IFN- $\gamma$ ) and inflammation inducers, lipopolysaccharides in rodents results in altered behaviors similar to those of depressed patients (Dantzer, O'Connor, Freund, Johnson, \& Kelley, 2008; Kentner et al., 2008). Whereas, cytokine antagonists, such as interleukin-1 (IL-1) receptor antagonist and prebiotics attenuate social and/or anxiety behavior in rodents (Arakawa, Blandino, \& Deak, 2009; Dantzer, 2004; Savignac et al., 2016).

Cytokines can be divided into several categories including IL, colony stimulating factor (CSF), IFN, tumor necrosis factor (TNF) and chemokine family. Circulating level of cytokins ranges according to the condition of the body. Importantly, cytokines respond to stress-triggered neuroinflammation, and modulate the function of neurons in coordination with astrocytes in the development of depression (Jia, Gao, \& Hu, 2020; C. Zhang et al., 2019). Various alterations of cytokines have been reported in patients with depression (Lotrich, 2015), including IL-1, IL-6, IL-2, IL-4, IL-10, IL-21, IL-22, TNF-a, TGF- $\beta$ and IFN- $\gamma$ (Kohler et al., 2018; Mosiolek et al., 2021; Nobis, Zalewski, \& Waszkiewicz, 2020). Besides, it was suggested that antidepressant treatment could decrease peripheral levels of IL-6, IL-10, TNF- $a$ and IFN- $\gamma$ (C. Y. Chen et al., 2018; Kohler et al., 2018).

Chronic mild stress (CMS) is a crucial trigger of depression (Paul Willner, 2017), and CMS-treated animals exhibit depression-like behaviors, such as helplessness and anhedonia (L. Chen et al., 2021; Garcia et al., 2009; X. Q. Wang et al., 2021). The objective of this study is to investigate the roles of NAl exposure in behavioral alterations induced by CMS treatment in mice. Contents of cortisol and cytokines in the serum were examined to explore possible contribution of immune responses and HPA axis in this process. We found that the NAI exposure could ameliorate CMS-induced depression-like behaviors shown by sucrose preference test and tail suspension test. In addition, the NAI exposure also interfered the alterations of cortisol levels and multiple cytokines in the serum, which may contribute to the behavioral changes in CMS-treated mice.

\section{Material And Methods}




\section{Animals}

Six-week-old male C57BL/6 mice were obtained from Shanghai Lingchang Biotechnology Co. Ltd, China. Animals were provided regular rodent chow and water ad libitum and were maintained on a $12 \mathrm{~h}$ light/dark cycle (lights on at 7:00 a.m.), with a temperature of $25 \pm 1^{\circ} \mathrm{C}$ and a humidity level of $50 \pm 10 \%$. Prior to the experimental procedure, mice were accommodated in the experiment room for two weeks. All procedures were carried out in compliance with the Animal Experimental Ethics Committee of Shanghai Medical School, Fudan University.

\section{Apparatus}

Filtered air (FA) box and NAI box (50 cm length $\times 40 \mathrm{~cm}$ width $\times 100 \mathrm{~cm}$ height) were equipped with a fan (ERF500D1N, Honeywell). The NAI box contained a negative ion generator purchased from Shanghai Sailumei Environmental Protection Technology Co., Ltd, China, and provided approximately $4 \times 10^{4}$ small NAls per cubic centimeter (high-density exposure) at 1 meter to the feeding cages. Automatic observation system of atmospheric negative ions (Wide Creative Science \& Technology, Beijing, China) was used to monitor the concentration of NAls whose ion mobility is not less than $0.4 \mathrm{~cm}^{2} /(\mathrm{V} \cdot \mathrm{s})$.

\section{Experimental procedure}

Mice were housed either in the FA box or NAI box. In each box, animals were randomly assigned into two groups: one suffered from CMS for 30 days and the other stayed as is. Therefore, the research was consisted of 4 groups: 1) Control mice in FA box, 2) CMS-treated mice in FA box, 3) Control mice in NAI box and 4) CMS-treated mice in NAI box. Mouse body weight between groups was evaluated before and after 30-day exposure. After the 30-day exposure, behavioral tests were performed and mice were sacrificed after completing these tests for examination of contents of cortisol and cytokines in the serum.

\section{CMS paradigms}

The CMS protocol was conducted according to the well-established methods with minor modifications (L. Chen et al., 2021; X. Q. Wang et al., 2021; Zhao et al., 2021). The mouse was restained in a 50-ml tube with no space to turn over for 6 hours every day, along with unpredictable 3-minute shaking for 5-7 times during this period.

\section{Behavioral testing}

Behavioral experiments were performed in a sound-proof room with a neutral environment. All behavioral tests were conducted during the light phase of the light/dark cycle. All mice were given a 30-minute habituation in the behavioral room before the start of each test. After each animal completed the behavioral test, the equipment was thoroughly cleaned to eliminate olfactory effects. The experimenter was blind to the group identity of the tested mice. Some behavioral tests were recorded by a video camera, then the footages were analyzed by a trained researcher.

\section{Open field test (OFT)}


A black square arena $(45 \times 45 \times 30 \mathrm{~cm})$ was used to examine locomotor activity. Mice were placed in the center of the arena and allowed to explore the apparatus freely for $5 \mathrm{~min}$ (Y. L. Wang et al., 2018). Total distance moving in the field was analyzed by the EthoVision XT video tracking software (Ver. 12).

\section{Sucrose preference test (SPT)}

All stages of the test were carried out at the same time of the day (C. Zhang et al., 2019). Each cage was provided with two drinking tubes containing sucrose water $(2 \% \mathrm{w} / \mathrm{v})$ during the first 24-hour training phase. Then the next day, one bottle with $2 \%$ sugar solution and another bottle with regular water were provided to mice. After training, mice were deprived of water and food for 24 hours, then the mice were given the free choice to drink from two bottles for 24 hours: one was filled with a sucrose solution, and the other was filled with water. The positions of the bottles in the cage were switched after the first 12 hours. Sucrose and water consumptions were recorded separately before and after the test. Sucrose preference $\%=($ sucrose intake/total intake $) \times 100 \%$. The total intake value is the sume of water intake value and sucrose intake value.

\section{Forced swimming test (FST)}

Animals were individually placed in a transparent acrylic cylinder (height $30 \mathrm{~cm}$, diameter $15 \mathrm{~cm}$ ) for 6 minutes (Zhao et al., 2021), which was filled with tap water to a depth of $20 \mathrm{~cm}$. The first 2 minutes were spent for adaptation, and the last 4 minutes were analyzed. Immobility time was evaluated as floating or no active movements except those necessary for the mouse to keep its head above water.

\section{Tail suspension test (TST)}

In the TST, mice were suspended $30 \mathrm{~cm}$ above the floor with an adhesive tape applied approximately 1 $\mathrm{cm}$ from the end of the tail on a metal hook (Xu et al., 2021). At the beginning of the test, nearly all the mice attempted to escape from hanging, but after a period of struggling, it showed intermittent immobility, displaying a state of "behavioral despair". The duration of this state was considered as the immobility time. The activities of the mice were recorded by a video camera, then the immobility time during the last 4 minutes of a 6-minute testing period was evaluated.

\section{Rotarod test (RTT)}

Mice were habituated to the rod for 2 minutes while it slowly rotated (10 rpm-rotations per minute) and they were replaced on the rod if they fell off during the 2 min. Testing consisted of three-trial sessions; each session had a progressively increasing speed from 4 rpm to 40 rpm within 5 minutes. On each of the three trials, the mouse was placed on the rod and left there until either 300 seconds elapsed or until the mouse fell off. There was a 1-hour break between trials. The average performance of total time on the rod for the three-trial session was analyzed.

\section{Y maze (YMT)}

Working memory was evaluated by spontaneous alternation Y-maze test (Pontifex et al., 2021). The apparatus comprised of three identical arms $(30 \mathrm{~cm} \times 5 \mathrm{~cm} \times 10 \mathrm{~cm})$, spaced $120^{\circ}$ apart. The mouse was 
placed in one arm of the maze and allowed to explore freely for 8 minutes. At the same time, zone transitionings were recorded by tracking software (EthoVision XT video tracking software, Ver. 12). Spontaneous alternation was calculated using the following formula: Spontaneous alternation $\%=$ (Number of alternations/Total Arm entries - 2) × 100 .

\section{Cytokine and cortisol measurement}

Blood was collecting from obital sinus in anesthetized mice. The serum from blood was isolated by centrifugation for $20 \mathrm{~min}$ at $1000 \times \mathrm{g}$ at $4^{\circ} \mathrm{C}$, then the aliquots of the samples were stored at $-80^{\circ} \mathrm{C}$ before they were measured. Cytokines, including granulocyte-colony stimulating factor (G-CSF), granulocytemacrophage colony-stimulating factor (GM-CSF), IL-1a, IL-1 $\beta$, IL-2, IL-3, IL-4, IL-5, IL-6, IL-7, IL-9, IL-10, IL12p70, IL-13, IL-15, IL-17, IL-21, IL-23, IFN- $y$ and TNF-a in the serum were determined using commercial Interleukin Antibody Arrays (QAM-INT-1, RayBiotech Inc). Serum concentration of cortisol was determined by using an enzyme linked immunosorbent assay (ELISA) kit (Enzo, ADI-900-071). Experiments were performed in accordance with the manufacturer's protocols.

\section{Statistical analysis}

All figures were performed using Graph Pad Prism 6 and IBM SPSS statistics 20.0. Data were expressed as the mean \pm standard error of mean (SEM). One-way analysis of variance (ANOVA) was used for statistical analysis of data, followed by Bonferroni (test of homogeneity of variance a is more than 0.05 ) or Tamhane's T2 (test of homogeneity of variance $a$ is less than 0.05 ) post-hoc multiple comparison test. Statistical significance was defined as $* P<0.05, * * P<0.01, * * * P<0.001$.

\section{Results}

\section{NAl exposure ameliorates CMS-induced depression-like behaviors}

During the 30-day experiment, the concentration of negative oxygen ions was monitored (Fig. 1), showing a consistent supply of NAls.

The CMS design and timeline of behavioral observations are shown in Fig. 2a. Depression-like behaviors were examined by the sucrose preference, force swimming and tail suspension tests. The reduction of preference to sucrose reflects the core depression-like behaviors in CMS-induced depression animal model (Czeh, Fuchs, Wiborg, \& Simon, 2016). As expected, the CMS treatment reduced the consumption of sucrose solution in FA group (Fig. 2b). Importantly, the negative ion intervention restored the sucrose preference in CMS-treated mice in NAl group compared with those in FA group (Fig. 2b). To further evaluate the effect of NAI on depression-like behaviors, TST and FST tests were performed. In the TST test, the CMS treatment led to significantly-increased immobility time in FA group, but did not do so in NAI group as shown by similar immobility time compared with control mice in NAI group (Fig. 2c). However, in the FST test, there weren't any differences revealed between control and CMS-treated mice in either FA or NAl group (Fig. 2d). In addition, short-term memory, a type of working memory responsibe for the 
temporary storage of information, (Czaczkes, 2018), was examined using $Y$ maze, and there were no differences found among the four groups (Fig. 2e).

It is well known that the CMS treatment prevents the increase of body weight during the one-month CMS period (Lu, Yang, Geng, Ding, \& Hu, 2014). The body weight before the experiment was not significantly different among the four groups (Fig. 2f). After 30-day experiment, in FA group, there was no weight gain in the CMS-treated mice, whereas control mice gained more weight than before (Fig. 2f, g). However, it should be noted that the unchanged body weight during the research in CMS-treated mice was also present in NAl group (Fig. 2f, g), showing that the NAl exposure has no effects on the alteration of body weight in CMS-treated mice, although it ameliorates CMS-induced depression-like behaviors as mentioned above.

To exclude the possibility that the behavioral alterations were associated with inability of locomotion activity, we carried out the rotarod test and OFT test, which are used to examine motor coordination and spontaneous locomotion (Moniruzzaman, Mannan, Hossen Khan, Abir, \& Afroze, 2018; Ramshini et al., 2018). It was found that there was no significant difference among the groups in the two tests in terms of time stayed on the rod and traveled distance in the open field (Fig. $2 \mathrm{~h}$, i). Taken together, we demonstrate that NAI exposure prevents the occurrence of "anhedonia" behavior and some aspects of "despair" behaviors induced by the CMS treatment.

\section{Effects of NAl exposure on cortisol levels of CMS-treated mice}

Hyperactivity of the HPA axis is one of well-documented factors in the etiology of depression, which can be reflected by the increased level of cortisol in serum (Dean \& Keshavan, 2017; Kim et al., 2016). Consistently, the CMS treatment did induce an increase of the concentrations of serous cortisol in FA group, but not in CMS-treated mice in NAI group (Fig. 3), showing that one-month NAI exposure relieves the HPA axis hyperactivity induced by CMS in mice.

\section{Effects of NAl exposure on cytokine levels of CMS-treated mice}

The CMS treatment increases the levels of corticosterone, which is known to negatively regulate immune responses (Villas Boas et al., 2019; Zaletel et al., 2016). To explore possible mechanism underlying the effect of NAl exposure on CMS-induced depression-like behaviors, we then measured the contents of a panel of cytokines in the serum, based on the concept that cytokines play important roles in immune responses as well as HPA axis activation (Kim et al., 2016). Comparing the data from control and CMStreated mice in FA group would be useful to evaluate if CMS treatment itself interferes the serous levels of cytokines. The results showed that the levels of IL-15 were up-regulated in CMS-treated mice, while those of IL-7 were down-regulated in the serum (Fig. 4a, b). Comparing the data from control mice in NAI and FA groups would provide the information if the NAl exposure itself has any contribution to alterations of cytokines, and it showed that the levels of IL-15 and IL-21 were increased, and those of IL-7 and TNF-a were decreased (Fig. 4a-d). In this study, a total of 20 cytokines were examined, and only a small number 
of them displays the changes in the serum, showing that specific cytokines are affected by the CMS or NAl exposure. The data showing unchanged levels of cytokines are included in Fig. S1.

Next, we asked if the NAI exposure contributes to the alteration of cytokines in CMS-treated mice. The increased levels of IL-15 were no longer existed, while the deceased levels of IL-7 were still present in CMS-treated mice with the NAI exposure, as compared with those without NAI exposure (Fig. 4a, b). There were increasing tendency of IL-13 and TNF-a levels in CMS-treated mice although the $p$ value was not statistically significant relative to control mice in FA group; while in NAl group, their levels of CMS-treated mice were reduced to the control levels (Fig. 4d, e). These results showed that the NAl exposure interferes the changes of serous cytokines in CMS-treated mice.

Leukocytes, especially T cell population plays a key role in immune responses, in which cytokines mediate intercellular communication. Th1 cells and Th2 cells are the two major subgroups of $\mathrm{T}$ cells that are characterized primarily on the basis of cytokines they secreted (Varade, Magadan, \& GonzalezFernandez, 2021). Th1 cells secret types I cytokines (e.g. IL-2 and IFN- $\gamma$ ) which are mainly pro-

inflammatory, while Th2 cells secret type II cytokines (e.g. IL-4, IL-5, IL-6, IL-10 and IL-13) which are mainly anti-inflammatory (Gharagozloo et al., 2013; Maher, Griffith, Lau, Reeves, \& Higgins, 2014). Accumulated evidence supports the idea that stress leads to an increase of pro-inflammatory cytokines (N. Li et al., 2016; S. Li et al., 2020). However, the data from animal models and clinical research revealed significant variabilities in type I and type II cytokine profiles (Cuervo, Sordillo, \& Abuelo, 2021; Koivisto et al., 2019; Razali et al., 2020). It seems to be clear that the examination of a single cytokine, or small groups of cytokines are not sufficient to evaluate the alterations of cytokines in depression-related animal models. Instead, one way to gain a better insight might be achieved by examining the ratios between the two types of cytokines, which reflects the balance between them and the tilt of immune response (Rostaing et al., 1999; Yoon, Kim, Lee, Kwon, \& Kim, 2012).

To this end, the ratios of type II (i.e., IL-4, IL-5, IL-6, IL-10 and IL-13) and type I cytokines (i.e., IL-2 and IFNY) were calculated. We found that these ratios of IL-4/IL-2, IL-5/IL-2 and IL-13/IL-2 were elevated in CMStreated mice, and remarkably the elevations of IL-4/IL-2 and IL-13/IL-2 were prevented by NAI exposure (Fig. 5a, c). There is also a decrease tendency of the ratio of IL-5/IL-2 after NAI exposure, although the $p$ value was not statistically significant (Fig. 5b). The others were not significantly different (Fig. S2). It can be concluded that the NAl exposure is likely having the ability of preventing the shift from Th1 to Th2 cytokine profiles in CMS-treated mice.

\section{Discussion}

In this study, we showed that NAI intervention can improve the depression-like behaviors in CMS-treated mice. We also demonstrated that the NAI intervention reduces the increase of serous cortisol levels and reestablish the balance between type I and type II cytokines in CMS-treated mice.

The core depressive symptom of CMS model is decreased sucrose preference in rodents (Czeh et al., 2016; P. Willner, 2005), and it was confirmed in our CMS-treated mice. The role of NAl exposure in 
alleviating depression-like behaviors are supported by the data of restoration of sucrose preference and reduction of immobility time in the TST in CMS-treated mice. Clinical data have shown that high-density negative air ionization is effective in treating seasonal affective disorders including major depression (Michael Terman, 2006; M. Terman, Terman, \& Ross, 1998). Based on these findings, it is recommended that NAI intervention is a useful tool or providing as an alternative way in treating depression. In addition to therapeutic application, it is also recommended that NAI exposure either in natural environment or by artificial way can be used in preventing the onset of mental illness for people who had experienced chronic/acute stress and/or displayed a trend of developing the disorders.

Glucocorticoids are the final products of the HPA axis, and regulates stress-triggered responses through a negative feedback with the hypothalamus and pituitary gland (van Bodegom, Homberg, \& Henckens, 2017). A wealth of evidence documented that chronic stress exposure leads to an impairment of the negative feedback of the HPA axis with increased levels of cortisol (Barfield \& Gourley, 2018; Kvarta, Bradbrook, Dantrassy, Bailey, \& Thompson, 2015). The hyperactivity of HPA axis is observed in the majority of depressed patients (Nemeroff \& Vale, 2005; Pruessner, Hellhammer, Pruessner, \& Lupien, 2003). In this study, we showed that the elevation of cortisol in the CMS-treated mice could be prevented by the NAI intervention, and this may be one of possible mechanisms underlying its role in ameliorating CMS-induced depression-like behaviors in mice.

Patients with depression often show signs of inflammation, which was illustrated by the increased concentrations of cytokines such as IL-1, IL-6, and TNF-a in the peripheral blood and cerebrospinal fluid (Leonard, 2007; Paudel, Shaikh, Shah, Kumari, \& Othman, 2018). It has been reported that administration of IFN-a, used to treat infective diseases, is a significant risk factor in inducing major depressive episode (Andrew H. Miller, Vladimir Maletic, \& Charles L. Raison, 2009; Su et al., 2019). Among 20 cytokines examined, two (IL-15 and IL-7) were altered in CMS-treated mice, and more (IL-7, IL-15, IL-21 and TNF-a) were changed by NAl exposure either in control mice or CMS-treated mice. To evaluate possible contribution of cytokines in the ameliorated depression-like behaviors by NAI intervention, we analyzed the alterations of ratios of proinflammatory and anti-inflammatory cytokines, which may provide a whole view concerning the net effects of altered cytokines in modulating brain functions of CMS-treated mice. The ratios of IL-4/IL-2, IL-5/IL-2 and IL-13/IL-2 were elevated in CMS-treated mice. After NAI treatment, two of them (IL-4/IL-2 and IL-13/IL-2) were reduced significantly similar to the level of control mice. The ratio of IL-5/IL-2 was also showing reduced tendency with no statistical difference. IL-2 functions as a type I cytokine, the alterations of IL-2-related ratios support the idea that CMS treatment may lead to the shift of Th1 inflammatory pathway with deterious effects on mood-related brain functions, whereas NAI intervention may prevent or reduce the activation whereby it displays beneficial effect in mood regulation.

Finally, preliminary data from patients with inflammatory disorders, as well as depressed patients without appearing any other medical problems, suggest that inhibiting inflammatory signaling pathways may improve depressed mood and increase effective treatment response to conventional antidepressant medication (Malemud \& Miller, 2008; J.-c. Zhang, Yao, \& Hashimoto, 2016). Translational implications of these findings include the unique opportunity to identify relevant patient populations, apply immune- 
targeted therapies, and monitor therapeutic efficacy at the level of the immune system in addition to behaviors.

\section{Conclusions}

In conclusion, chronic stress results in depressive-like behaviors and has a significant effect on HPA axis and immune system parameters, which are the risk factors in development of depression. We demonstrated that NAI exposure is capable of preventing depression-like behaviors induced by chronic stress, and this positive effect is likely achieved by rebalancing activity of HPA axis and immuneresponse under the condition of chronic stress.

\section{Declarations}

\section{Availability of data}

All data generated or analysed during this study are included in this published article and its supplementary information files. The datasets used and/or analyzed during the current study are available from the corresponding author on reasonable request.

\section{Author information}

\section{Affiliations}

State Key Laboratory of Medical Neurobiology and MOE Frontiers Center for Brain Science, Institutes of Brain Science, Fudan University, Shanghai 200032, China

Yunqing Hu \& Yuqiang Ding

Department of Atmospheric and Oceanic Sciences \& Institute of Atmospheric Sciences, Fudan University, Shanghai, China

Ji Zhou

Department of Laboratory Animal Science, Fudan University, Shanghai 200032, China

Ying Huang \& Yuqiang Ding

Institute of Occupational Health and Environmental Health, School of Public Health, Lanzhou University, Lanzhou, Gansu 730000, China

Tingting Niu

Shanghai Typhoon Institute, CMA, Shanghai 200030, China Jianming Xu, Li Peng \& Ji Zhou 


\section{Shanghai Key Laboratory of Meteorology and Health, Shanghai Meteorological Bureau, Shanghai 200030, China}

Jianming Xu, Li Peng \& Ji Zhou

School of Public Health, Joint China-US Research Center for Environment and Pulmonary Diseases, Zhejiang Chinese Medical University, Hangzhou 310053, China

Qinghua Sun

Contributions

YQD and JZ design this work. YQH and TTN performed the animal model and histological examination. The first draft of the manuscript was written by $Y Q H$ and all authors commented on previous versions of the manuscript. JMX, LP, QHS analyzed and interpreted the research data. YH finally review and edit the manuscript. All authors read and approved the final manuscript.

Corresponding author

Correspondence to Ji Zhou and Yuqiang Ding.

Ethics declarations

Ethics approval

All procedures were carried out in compliance with the Animal Experimental Ethics Committee of Shanghai Medical School, Fudan University.

Consent for publication

Not applicable.

Competing interests

The authors declare no competing interests.

Funding

Not applicable.

\section{References}

1. Arakawa $H$, Blandino P, Deak T (2009) Central infusion of interleukin-1 receptor antagonist blocks the reduction in social behavior produced by prior stressor exposure. Physiol Behav 98(1-2):139-146. doi:10.1016/j.physbeh.2009.04.024 
2. Bachman CH, McDonald RD, Lorenz PJ (1966) Some effects of air ions on the activity of rats. Int J Biometeorol 10(1):39-46. doi:10.1007/bf01636119

3. Bailey WH, Williams AL, Leonhard MJ (2018) Exposure of laboratory animals to small air ions: a systematic review of biological and behavioral studies. Biomed Eng Online 17(1):72. doi:10.1186/s12938-018-0499-z

4. Barfield ET, Gourley SL (2018) Prefrontal cortical trkB, glucocorticoids, and their interactions in stress and developmental contexts. Neurosci Biobehav Rev 95:535-558. doi:10.1016/j.neubiorev.2018.10.015

5. Capuron L, Miller AH (2011) Immune system to brain signaling: neuropsychopharmacological implications. Pharmacol Ther 130(2):226-238. doi:10.1016/j.pharmthera.2011.01.014

6. Cernackova A, Durackova Z, Trebaticka J, Mravec B (2020) Neuroinflammation and depressive disorder: The role of the hypothalamus. J Clin Neurosci 75:5-10. doi:10.1016/j.jocn.2020.03.005

7. Chen CY, Yeh YW, Kuo SC, Liang CS, Ho PS, Huang CC,... Huang SY (2018) Differences in immunomodulatory properties between venlafaxine and paroxetine in patients with major depressive disorder. Psychoneuroendocrinology 87:108-118. doi:10.1016/j.psyneuen.2017.10.009

8. Chen L, Gong WK, Yang CP, Shao CC, Song NN, Chen JY,.. . Ding YQ (2021) Pten is a key intrinsic factor regulating raphe $5-\mathrm{HT}$ neuronal plasticity and depressive behaviors in mice. Transl Psychiatry 11(1):186. doi:10.1038/s41398-021-01303-z

9. Chu $\mathrm{CH}$, Chen SR, Wu CH, Cheng YC, Cho YM, Chang YK (2019) The effects of negative air ions on cognitive function: an event-related potential (ERP) study. Int J Biometeorol 63(10):1309-1317. doi:10.1007/s00484-019-01745-7

10. Cuervo W, Sordillo LM, Abuelo A (2021) Oxidative Stress Compromises Lymphocyte Function in Neonatal Dairy Calves. Antioxidants, 10(2). doi:10.3390/antiox10020255

11. Czaczkes TJ (2018) Using T- and Y-mazes in myrmecology and elsewhere: a practical guide. Insectes Soc 65(2):213-224. doi:10.1007/s00040-018-0621-z

12. Czeh B, Fuchs E, Wiborg O, Simon M (2016) Animal models of major depression and their clinical implications. Prog Neuropsychopharmacol Biol Psychiatry 64:293-310. doi:10.1016/j.pnpbp.2015.04.004

13. Dantzer R (2004) Cytokine-induced sickness behaviour: a neuroimmune response to activation of innate immunity. Eur J Pharmacol 500(1-3):399-411. doi:10.1016/j.ejphar.2004.07.040

14. Dantzer R, O'Connor JC, Freund GG, Johnson RW, Kelley KW (2008) From inflammation to sickness and depression: when the immune system subjugates the brain. Nat Rev Neurosci 9(1):46-56. doi:10.1038/nrn2297

15. Dean J, Keshavan M (2017) The neurobiology of depression: An integrated view. Asian J Psychiatr 27:101-111. doi:10.1016/j.ajp.2017.01.025

16. Della Vecchia A, Mucci F, Marazziti D (2020) Negative air ions in neuropsychiatry: a novel therapeutic option? Eur Neuropsychopharmacol 40:S167-S168. doi:10.1016/j.euroneuro.2020.09.219 
17. Duman RS, Aghajanian GK, Sanacora G, Krystal JH (2016) Synaptic plasticity and depression: new insights from stress and rapid-acting antidepressants. Nat Med 22(3):238-249. doi: $10.1038 / \mathrm{nm} .4050$

18. Garcia LSB, Comim CM, Valvassori SS, Reus GZ, Stertz L, Kapczinski F,.. . Quevedo J (2009) Ketamine treatment reverses behavioral and physiological alterations induced by chronic mild stress in rats. Progress in Neuro-Psychopharmacology Biological Psychiatry 33(3):450-455. doi:10.1016/j.pnpbp.2009.01.004

19. Gharagozloo M, Jafari S, Esmaeil N, Javid EN, Bagherpour B, Rezaei A (2013) Immunosuppressive effect of silymarin on mitogen-activated protein kinase signalling pathway: the impact on $\mathrm{T}$ cell proliferation and cytokine production. Basic Clin Pharmacol Toxicol 113(3):209-214. doi:10.1111/bcpt.12088

20. Gold PW (2015) The organization of the stress system and its dysregulation in depressive illness. Mol Psychiatry 20(1):32-47. doi:10.1038/mp.2014.163

21. Himmerich H, Patsalos O, Lichtblau N, Ibrahim MAA, Dalton B (2019) Cytokine Research in Depression: Principles, Challenges, and Open Questions. Front Psychiatry 10:30. doi:10.3389/fpsyt.2019.00030

22. Hinkelmann K, Moritz S, Botzenhardt J, Riedesel K, Wiedemann K, Kellner M, Otte C (2009) Cognitive Impairment in Major Depression: Association with Salivary Cortisol. Biol Psychiat 66(9):879-885. doi:10.1016/j.biopsych.2009.06.023

23. Jia X, Gao Z, Hu H (2020) Microglia in depression: current perspectives. Sci China Life Sci. doi:10.1007/s11427-020-1815-6

24. Jiang SY, Ma A, Ramachandran S (2018) Negative Air lons and Their Effects on Human Health and Air Quality Improvement. Int J Mol Sci, 19(10). doi:10.3390/ijms19102966

25. Kentner AC, Takeuchi A, James JS, Miki T, Seino S, Hayley S, Bielajew C (2008) The effects of rewarding ventral tegmental area stimulation and environmental enrichment on lipopolysaccharideinduced sickness behavior and cytokine expression in female rats. Brain Res 1217:50-61. doi:10.1016/j.brainres.2008.04.041

26. Kim YK, Na KS, Myint AM, Leonard BE (2016) The role of pro-inflammatory cytokines in neuroinflammation, neurogenesis and the neuroendocrine system in major depression. Prog Neuropsychopharmacol Biol Psychiatry 64:277-284. doi:10.1016/j.pnpbp.2015.06.008

27. Kohler CA, Freitas TH, Stubbs B, Maes M, Solmi M, Veronese N,.. . Carvalho AF (2018) Peripheral Alterations in Cytokine and Chemokine Levels After Antidepressant Drug Treatment for Major Depressive Disorder: Systematic Review and Meta-Analysis. Mol Neurobiol 55(5):4195-4206. doi:10.1007/s12035-017-0632-1

28. Koivisto AE, Olsen T, Paur I, Paulsen G, Bastani NE, Garthe I,.. . Bohn SK (2019) Effects of antioxidantrich foods on altitude-induced oxidative stress and inflammation in elite endurance athletes: $A$ randomized controlled trial. Plos One, 14(6). doi:10.1371/journal.pone.0217895 
29. Krishnan V, Nestler EJ (2008) The molecular neurobiology of depression. Nature 455(7215):894902. doi:10.1038/nature07455

30. Kvarta MD, Bradbrook KE, Dantrassy HM, Bailey AM, Thompson SM (2015) Corticosterone mediates the synaptic and behavioral effects of chronic stress at rat hippocampal temporoammonic synapses. J Neurophysiol 114(3):1713-1724. doi:10.1152/jn.00359.2015

31. Leonard BE (2007) Inflammation, depression and dementia: are they connected? Neurochem Res 32(10):1749-1756. doi:10.1007/s11064-007-9385-y

32. Leonard BE (2018) Inflammation and depression: a causal or coincidental link to the pathophysiology? Acta Neuropsychiatr 30(1):1-16. doi:10.1017/neu.2016.69

33. Li N, Wang X-M, Jiang L-J, Zhang M, Li N, Wei Z-Z,... Zhao Y-J (2016) Effects of endoplasmic reticulum stress on the expression of inflammatory cytokines in patients with ulcerative colitis. World J Gastroenterol 22(7):2357-2365. doi:10.3748/wjg.v22.i7.2357

34. Li S, Li J, Liu Y, Li C, Zhang R, Bao J (2020) Effects of Intermittent Mild Cold Stimulation on mRNA Expression of Immunoglobulins, Cytokines, and Toll-Like Receptors in the Small Intestine of Broilers. Animals, 10(9). doi:10.3390/ani10091492

35. Lotrich FE (2015) Inflammatory cytokine-associated depression. Brain Res 1617:113-125. doi:10.1016/j.brainres.2014.06.032

36. Lu M, Yang JZ, Geng F, Ding JH, Hu G (2014) Iptakalim confers an antidepressant effect in a chronic mild stress model of depression through regulating neuro-inflammation and neurogenesis. Int $\mathrm{J}$ Neuropsychopharmacol 17(9):1501-1510. doi:10.1017/S1461145714000285

37. Maher IE, Griffith JE, Lau Q, Reeves T, Higgins DP (2014) Expression profiles of the immune genes CD4, CD8beta, IFNgamma, IL-4, IL-6 and IL-10 in mitogen-stimulated koala lymphocytes (Phascolarctos cinereus) by qRT-PCR. PeerJ 2:e280. doi:10.7717/peerj.280

38. Malemud CJ, Miller AH (2008) Pro-inflammatory cytokine-induced SAPK/MAPK and JAK/STAT in rheumatoid arthritis and the new anti-depression drugs. Expert Opinion on Therapeutic Targets 12(2):171-183. doi:10.1517/14728222.12.2.171

39. Miller AH, Maletic V, Raison CL (2009) Inflammation and its discontents: the role of cytokines in the pathophysiology of major depression. Biol Psychiatry 65(9):732-741.

doi:10.1016/j.biopsych.2008.11.029

40. Miller AH, Maletic V, Raison CL (2009) Inflammation and Its Discontents: The Role of Cytokines in the Pathophysiology of Major Depression. Biol Psychiat 65(9):732-741. doi:10.1016/j.biopsych.2008.11.029

41. Moniruzzaman M, Mannan MA, Hossen Khan MF, Abir AB, Afroze M (2018) The leaves of Crataeva nurvala Buch-Ham. modulate locomotor and anxiety behaviors possibly through GABAergic system. BMC Complement Altern Med 18(1):283. doi:10.1186/s12906-018-2338-y

42. Mosiolek A, Pieta A, Jakima S, Zborowska N, Mosiolek J, Szulc A (2021) Effects of Antidepressant Treatment on Peripheral Biomarkers in Patients with Major Depressive Disorder (MDD). J Clin Med, 10(8). doi:10.3390/jcm10081706 
43. Nemeroff, C. B., \& Vale, W. W. (2005). The neurobiology of depression: Inroads to treatment and new drug discovery. Journal of Clinical Psychiatry, 66, 5-13. Retrieved from $<\mathrm{Go}$ to ISI>://WOS:000232011600002

44. Nikkheslat N, Pariante CM, Zunszain PA (2018) Neuroendocrine Abnormalities in Major Depression: An Insight Into Glucocorticoids, Cytokines, and the Kynurenine Pathway

45. Nobis A, Zalewski D, Waszkiewicz N (2020) Peripheral Markers of Depression. J Clin Med, 9(12). doi:10.3390/jcm9123793

46. Olivereau JM, Lambert JF, Truongngoc A (1981) INFLUENCE OF AIR IONS ON BRAIN ACTIVITY INDUCED BY ELECTRICAL-STIMULATION IN THE RAT. Int J Biometeorol, 25(1), 63-69. doi:10.1007/bf02184440

47. Paudel YN, Shaikh MF, Shah S, Kumari Y, Othman I (2018) Role of inflammation in epilepsy and neurobehavioral comorbidities: Implication for therapy. Eur J Pharmacol 837:145-155. doi:10.1016/j.ejphar.2018.08.020

48. Perez V, Alexander DD, Bailey WH (2013) Air ions and mood outcomes: a review and meta-analysis. BMC Psychiatry, 13. doi:Artn 29

49. $1186 / 1471-244 x-13-29$

50. Pontifex MG, Martinsen A, Saleh RNM, Harden G, Tejera N, Muller M,.. . Minihane AM (2021) APOE4 genotype exacerbates the impact of menopause on cognition and synaptic plasticity in APOE-TR mice. FASEB J 35(5):e21583. doi:10.1096/fj.202002621RR

51. Pruessner M, Hellhammer DH, Pruessner JC, Lupien SJ (2003) Self-reported depressive symptoms and stress levels in healthy young men: Associations with the cortisol response to awakening. Psychosom Med 65(1):92-99. doi:10.1097/01.Psy.0000040950.22044.10

52. Ramshini E, Sepehri G, Ahmadi-Zeidabadi M, Arjmand S, Khaksari M, Shabani M (2018) Contribution of CB1Rs in anxiety-related behaviors but not locomotor deficits induced by methamphetamine. Neurosci Lett 665:240-245. doi:10.1016/j.neulet.2017.12.021

53. Razali N, Hohjoh H, Inazumi T, Maharjan BD, Nakagawa K, Konishi M,.. . Hasegawa H (2020) Induced Prostanoid Synthesis Regulates the Balance between Th1-and Th2-Producing Inflammatory Cytokines in the Thymus of Diet-Restricted Mice. Biological Pharmaceutical Bulletin 43(4):649-662. doi:10.1248/bpb.b19-00838

54. Rostaing, L., Tkaczuk, J., Durand, M., Peres, C., Durand, D., de Preval, C.,.. . Abbal, M. (1999). Kinetics of intracytoplasmic Th1 and Th2 cytokine production assessed by flow cytometry following in vitro activation of peripheral blood mononuclear cells. Cytometry, 35(4), 318-328. doi:Doi 10.1002/(Sici)1097-0320(19990401)35:4<318::Aid-Cyto4>3.0.Co;2-4

55. Savignac HM, Couch Y, Stratford M, Bannerman DM, Tzortzis G, Anthony DC, Burnet PWJ (2016) Prebiotic administration normalizes lipopolysaccharide (LPS)-induced anxiety and cortical 5-HT2A receptor and IL1-beta levels in male mice. Brain Behav Immun 52:120-131.

doi:10.1016/j.bbi.2015.10.007 
56. Su K-P, Lai H-C, Peng C-Y, Su W-P, Chang JP-C, Pariante CM (2019) Interferon-alpha-induced depression: Comparisons between early- and late-onset subgroups and with patients with major depressive disorder. Brain Behavior Immunity 80:512-518. doi:10.1016/j.bbi.2019.04.032

57. Terman M (2006) Controlled Trial of Naturalistic Dawn Simulation and Negative Air lonization for Seasonal Affective Disorder. Am J Psychiatry, 163(12). doi:10.1176/ajp.2006.163.12.2126

58. Terman M, Terman JS, Ross DC (1998) A controlled trial of timed bright light and negative air ionization for treatment of winter depression. Arch Gen Psychiatry 55(10):875-882. doi:10.1001/archpsyc.55.10.875

59. van Bodegom M, Homberg JR, Henckens MJAG (2017) Modulation of the Hypothalamic-PituitaryAdrenal Axis by Early Life Stress Exposure. Front Cell Neurosci, 11. doi:10.3389/fncel.2017.00087

60. Varade J, Magadan S, Gonzalez-Fernandez A (2021) Human immunology and immunotherapy: main achievements and challenges. Cell Mol Immunol 18(4):805-828. doi:10.1038/s41423-020-00530-6

61. Villas Boas GR, de Lacerda B, Paes R, Gubert MM, Almeida P, Rescia W, Oesterreich VC, S. A (2019) Molecular aspects of depression: A review from neurobiology to treatment. Eur J Pharmacol 851:99121. doi:10.1016/j.ejphar.2019.02.024

62. Wang XQ, Zhang L, Xia ZY, Chen JY, Fang Y, Ding YQ (2021) PTEN in prefrontal cortex is essential in regulating depression-like behaviors in mice. Transl Psychiatry 11(1):185. doi:10.1038/s41398-02101312-y

63. Wang YL, Han QQ, Gong WQ, Pan DH, Wang LZ, Hu W,.. . Liu Q (2018) Microglial activation mediates chronic mild stress-induced depressive- and anxiety-like behavior in adult rats. J Neuroinflammation 15(1):21. doi:10.1186/s12974-018-1054-3

64. Willner $P$ (2005) Chronic mild stress (CMS) revisited: consistency and behavioural-neurobiological concordance in the effects of CMS. Neuropsychobiology 52(2):90-110. doi:10.1159/000087097

65. Willner $P$ (2017) The chronic mild stress (CMS) model of depression: History, evaluation and usage. Neurobiology of Stress 6:78-93. doi:10.1016/j.ynstr.2016.08.002

66. Xu GJ, Zhang Q, Li SY, Zhu YT, Yu KW, Wang CJ,.. . Wu Y (2021) Environmental enrichment combined with fasudil treatment inhibits neuronal death in the hippocampal CA1 region and ameliorates memory deficits. Neural Regen Res 16(8):1460-1466. doi:10.4103/1673-5374.303034

67. Yoon HK, Kim YK, Lee HJ, Kwon DY, Kim L (2012) Role of cytokines in atypical depression. Nord J Psychiatry 66(3):183-188. doi:10.3109/08039488.2011.611894

68. Zaletel I, Filipovic D, Puskas N (2016) Chronic stress, hippocampus and parvalbumin-positive interneurons: what do we know so far? Rev Neurosci 27(4):397-409. doi:10.1515/revneuro-20150042

69. Zhang C, Zhang YP, Li YY, Liu BP, Wang HY, Li KW,.. . Song C (2019) Minocycline ameliorates depressive behaviors and neuro-immune dysfunction induced by chronic unpredictable mild stress in the rat. Behav Brain Res 356:348-357. doi:10.1016/j.bbr.2018.07.001

70. Zhang J-c, Yao W, Hashimoto K (2016) Brain-derived Neurotrophic Factor (BDNF)-TrkB Signaling in Inflammation-related Depression and Potential Therapeutic Targets. Curr Neuropharmacol 
14(7):721-731. doi:10.2174/1570159x14666160119094646

71. Zhao Y, Coulson EJ, Su X, Zhang J, Sha B, Xu H,.. . Wang S (2021) Identification of 14-3-3 epsilon as a regulator of the neural apoptotic pathway for chronic-stress-induced depression. iScience 24(2):102043. doi:10.1016/j.isci.2021.102043

72. Zunszain PA, Anacker C, Cattaneo A, Carvalho LA, Pariante CM (2011) Glucocorticoids, cytokines and brain abnormalities in depression. Prog Neuropsychopharmacol Biol Psychiatry 35(3):722-729. doi:10.1016/j.pnpbp.2010.04.011

Figures

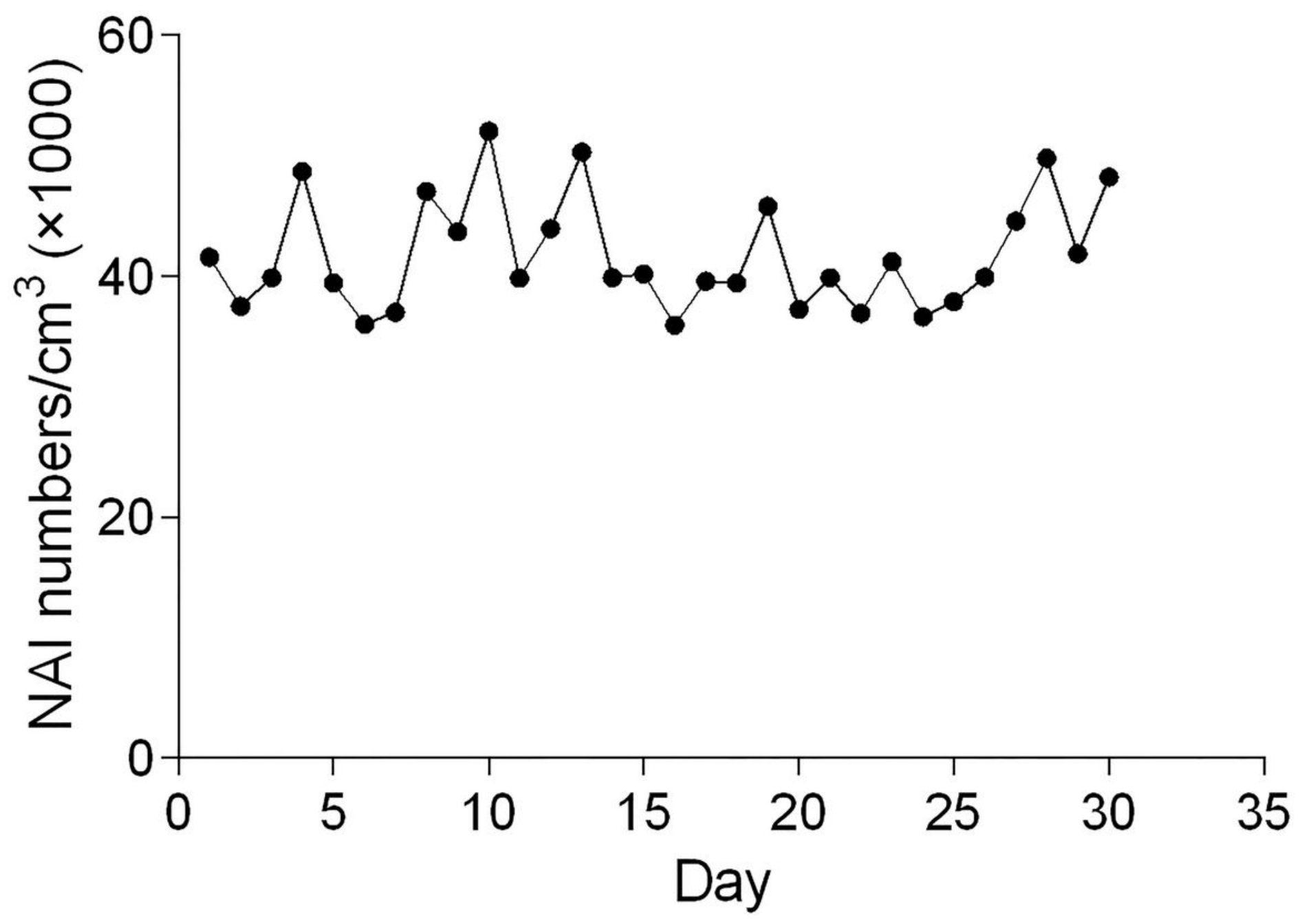

Figure 1

The concentration of negative air ions detected daily 

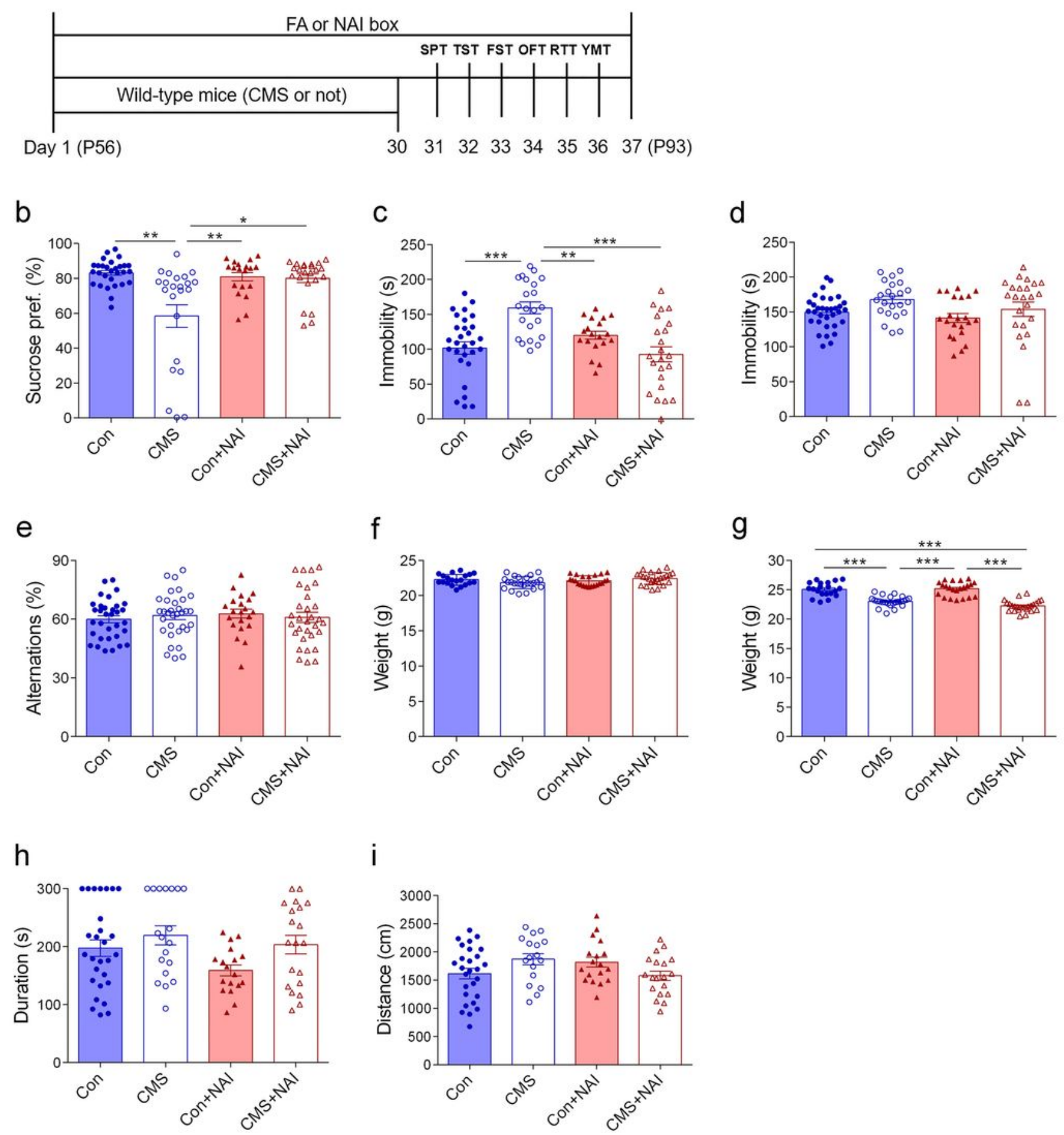

\section{Figure 2}

NAl intervention alleviates depression-like behavior in mice after stress exposure (a) Diagram of experiment design and timeline. (b) Sucrose preference in the SPT. The one-way ANOVA test measured

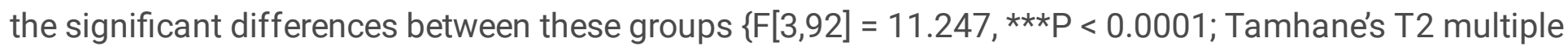
comparisons test showed ${ }^{* *} \mathrm{P}=0.003$ (Con vs. CMS); $\mathrm{P}=0.012$ (CMS vs. CMS+NAI); ${ }^{* \star} \mathrm{P}=0.009$ (CMS vs. Con+NAI)\}. (c) Time spent immobile in the TST. The one-way ANOVA test measured the significant 
differences between these groups $\{F[3,90]=10.431$, $* \star * P<0.0001$; Tamhane's T2 multiple comparisons

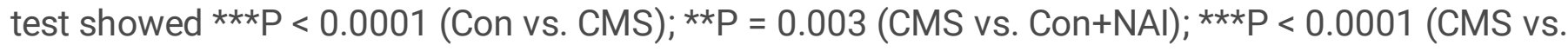
CMS+NAI) \}. (d) Time spent immobile in the FST. The one-way ANOVA test with Tamhane's T2 multiple comparisons test measured the significant differences between these groups $\{F[3,98]=2.475, P=0.066\}$. (e) Spontaneous alternation in Y maze. The one-way ANOVA test with Bonferroni multiple comparisons test measured the significant differences between these groups $\{F[3,122]=0.366, P=0.778\}$. (f) Body weight evaluated before the experiment. The one-way ANOVA test with Bonferroni multiple comparisons test measured the significant differences between these groups $\{F[3,87]=2.509, P=0.064\}$. $(\mathrm{g})$ Body weight evaluated after 30-day CMS exposure. The one-way ANOVA test measured the significant differences between these groups $\{F[3,87]=45.286, * \star * P<0.0001$; Bonferroni multiple comparisons test

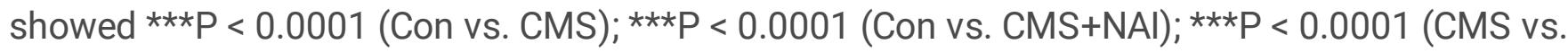
Con+NAl); ${ }^{* \star *} \mathrm{P}<0.0001$ (CMS+NAl vs. Con+NAI) \}. (h) Time spent on the rod. The one-way ANOVA test with Tamhane's T2 multiple comparisons test measured the significant differences between these groups $\{F[3,81]=2.681, P=0.052\}$. (i) Distance traveled in the open field. The one-way ANOVA test with Bonferroni multiple comparisons test measured the significant differences between these groups $\{F[3,77]$ $=2.338, \mathrm{P}=0.08\} .{ }^{\star} \mathrm{P}<0.05, * * \mathrm{P}<0.01,{ }^{\star \star *} \mathrm{P}<0.001$. Data are presented as mean \pm S.E.M. 


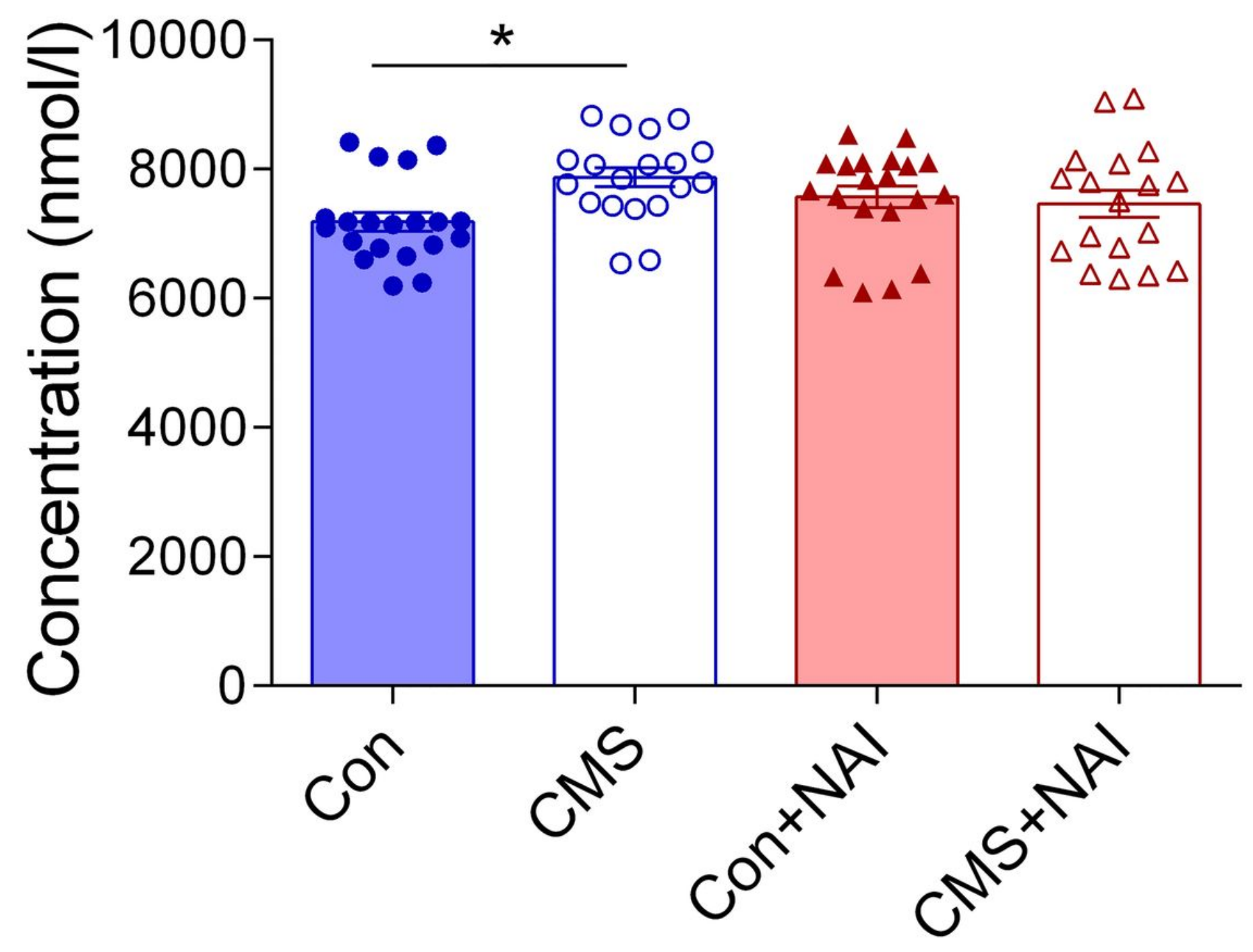

Figure 3

Effects of NAI intervention on cortisol levels in serum of mice with CMS. A significant difference is observed between control (Con) and CMS-treated (CMS) mice in FA group but not NAI group. The one-way ANOVA test measured the significant differences between these groups $\left\{F[3,73]=2.924\right.$, ${ }^{*} P=0.039$; Bonferroni multiple comparisons test showed $* \mathrm{P}=0.027$ (Con vs. CMS) $\}$. 
a

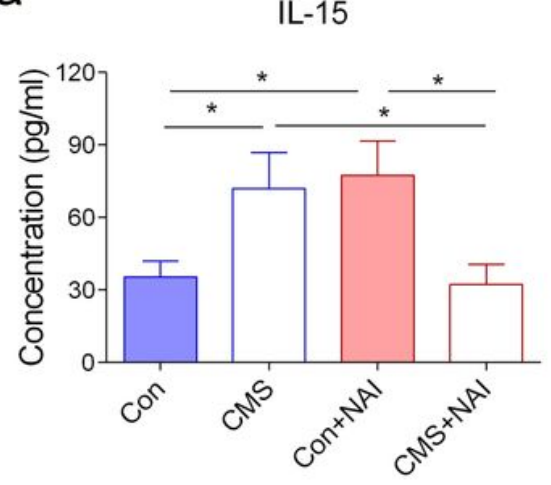

d

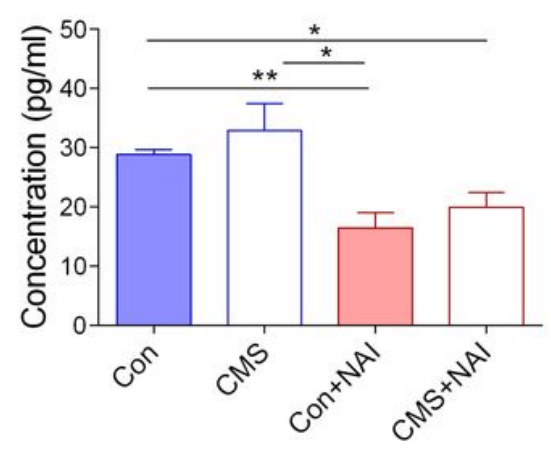

b

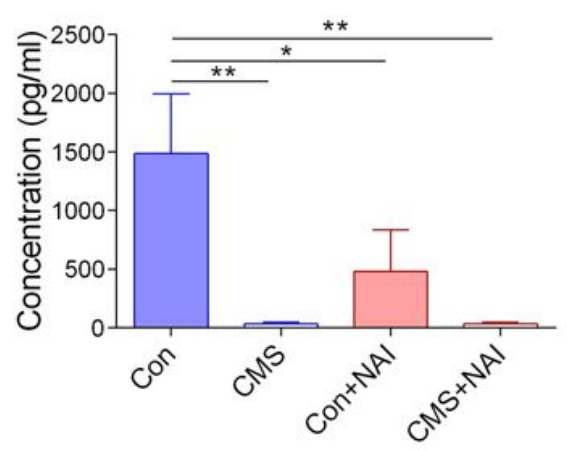

e

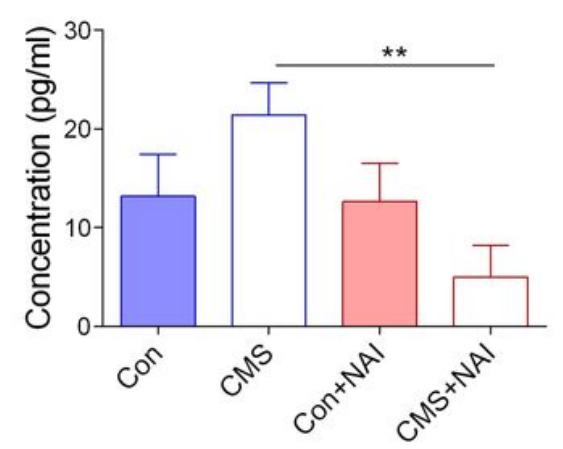

C

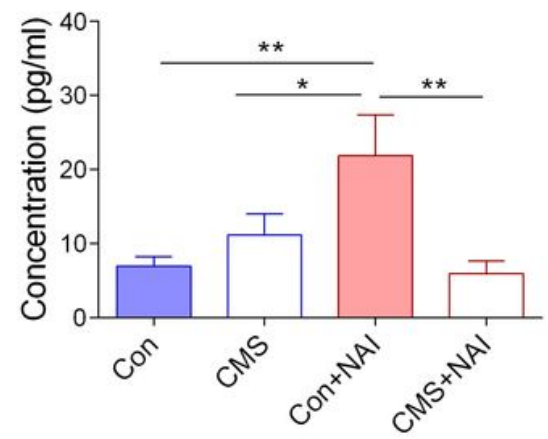

$f$

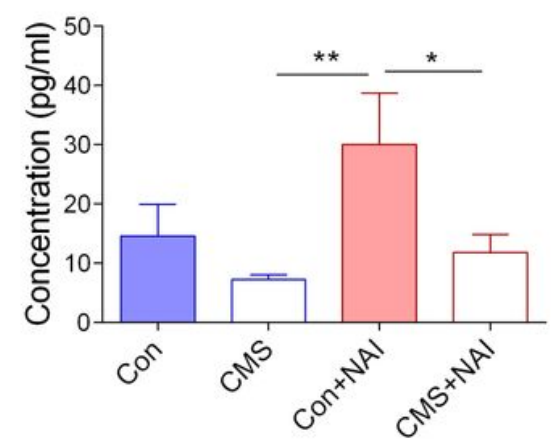

Figure 4

Effects of NAl innervation on inflammatory cytokine levels in serum of mice with CMS (a) The protein concentration of IL-15. The one-way ANOVA test measured the significant differences between these groups $\left\{F[3,35]=4.055,{ }^{*} P=0.014\right.$; Bonferroni multiple comparisons test showed ${ }^{*} P=0.033$ (CMS vs. Con); ${ }^{*} \mathrm{P}=0.015$ (Con+NAl vs. Con); ${ }^{*} \mathrm{P}=0.024$ (CMS vs. CMS+NAI); ${ }^{*}=0.011$ (CMS+NAI vs. Con+NAI) . (b) The protein concentration of IL-7. The one-way ANOVA test measured the significant differences between these groups $\{\mathrm{F}[3,34]=4.485$, $* * \mathrm{P}=0.009$; Bonferroni multiple comparisons test showed $\mathrm{P}=$ $\star \star 0.003$ (CMS vs. Con); $* \star P=0.003$ (CMS+NAl vs. Con); $* \mathrm{P}=0.032$ (Con vs. Con+NAI) $\}$. (c) The protein concentration of IL-21. The one-way ANOVA test measured the significant differences between these groups $\left\{F[3,35]=4.703, P=\star \star 0.007\right.$; Bonferroni multiple comparisons test showed ${ }^{*} \mathrm{P}=0.004$ (Con vs. Con+NAI); ${ }^{*}=0.029$ (CMS vs. Con+NAI); ** $=0.002(\mathrm{CMS}+\mathrm{NAI}$ vs. Con+NAI) $\}$. (d) The protein concentration of TNF- $a$. The one-way ANOVA test measured the significant differences between these groups $\left\{F[3,35]=6.485\right.$, $* * P=0.001$; Tamhane's $T 2$ multiple comparisons test showed ${ }^{*} P=0.033$ $\left(C M S+N A l\right.$ vs. Con); ${ }^{* *} \mathrm{P}=0.005$ (Con vs. Con+NAI); ${ }^{*} \mathrm{P}=0.044$ (CMS vs. Con+NAI) $\}$. (e) The protein concentration of IL-13. The one-way ANOVA test measured the significant differences between these groups $\left\{F[3,34]=3.328, * P=0.031\right.$; Bonferroni multiple comparisons test showed ${ }^{*} P=0.003$ (CMS vs. CMS+NAI) \}. (f) The protein concentration of IL-6. The one-way ANOVA test measured the significant differences between these groups $\{F[3,33]=3.304, * P=0.032$; Bonferroni multiple comparisons test 
showed ${ }^{\star *} P=0.006\left(C M S\right.$ vs. Con+NAl); ${ }^{*} P=0.025(C M S+N A l$ vs. Con+NAl) $\} .\left({ }^{*} P<0.05,{ }^{\star *} P<0.01\right)$. $9 \leq \mathrm{n} \leq 11 /$ group.
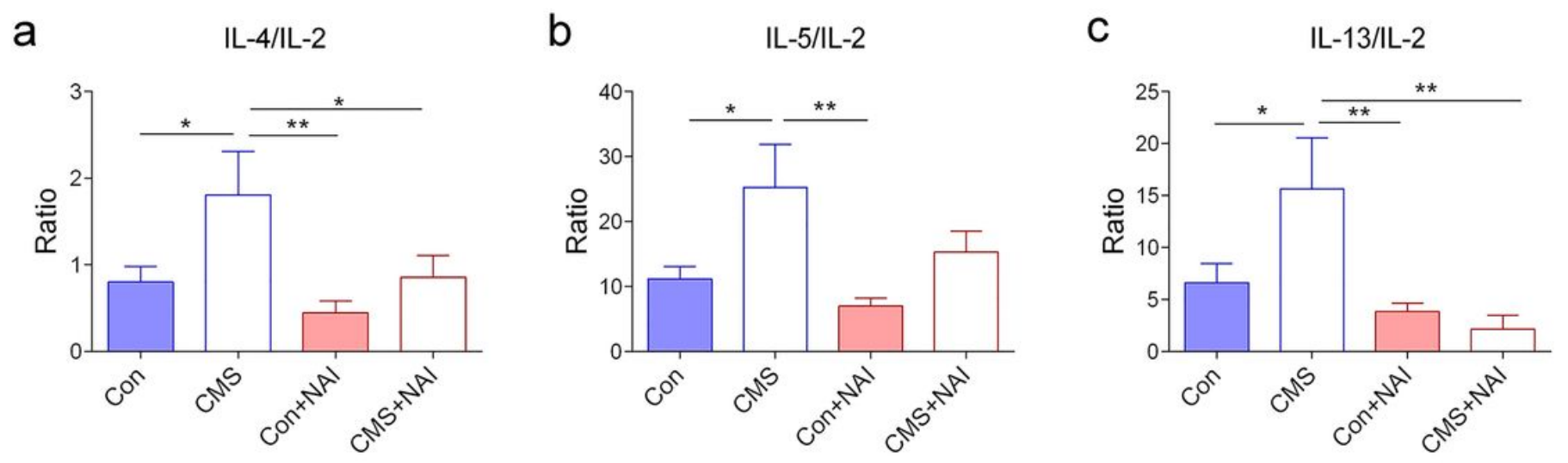

\section{Figure 5}

Comparison of ratios of IL-4/IL-2, IL-5/IL-2 and IL-13/IL-2 in CMS-treated mice with and without NAI exposure (a) The ratio of serous concentration of IL-4/IL-2. The one-way ANOVA test measured the significant differences between these groups $\{F[3,35]=3.599, * P=0.023$; Bonferroni multiple comparisons test showed $* \mathrm{P}=0.030$ (CMS vs. Con); ${ }^{*} \mathrm{P}=0.035$ (CMS vs. $\left.\mathrm{CMS}+\mathrm{NAI}\right)$; ${ }^{*} \mathrm{P}=0.003$ (CMS vs. Con+NAI) \}. (b) The ratio of serous concentration of IL-5/IL-2. The one-way ANOVA test measured the significant differences between these groups $\{F[3,35]=3.981$, * $P=0.015$; Bonferroni multiple comparisons test showed $* P=0.018$ (CMS vs. Con); $P=\star \star 0.002$ (Con+NAl vs. CMS) $\}$. (c) The ratio of serous concentration of IL-13/IL-2. The one-way ANOVA test measured the significant differences between these groups $\{F[3,33]=5.016, P=0.006$; Bonferroni multiple comparisons test showed $* P=$ 0.024 (CMS vs. Con); ${ }^{\star *} \mathrm{P}=0.001$ (CMS+NAl vs. CMS); ${ }^{\star \star} \mathrm{P}=0.003$ (CMS vs. Con+NAI) $\} .\left({ }^{*} \mathrm{P}<0.05,{ }^{*} \mathrm{P}<\right.$ $0.01) .9 \leq n \leq 11 /$ group.

\section{Supplementary Files}

This is a list of supplementary files associated with this preprint. Click to download.

- SI.pdf 